\title{
A clinical study on the treatment of granulomatous lobular mastitis by the external application of the internal pus-expelling decoction and operation
}

\author{
Peng-Zhou Liu ${ }^{1}$, Xiao-Guang Shi ${ }^{2}$, Xi-Meng Zuo ${ }^{1}$, Rui Lai ${ }^{1}$, Ze Zhao ${ }^{1}$, Zhen-Rui Yang ${ }^{1}$, Jie-Li Liu ${ }^{1}$ \\ ${ }^{1}$ Beijing University of Chinese Medicine, Beijing, China; ${ }^{2}$ Department of Galactophore Dongzhimen Hospital of Beijing University of Chinese \\ Medicine, Beijing, China \\ Contributions: (I) Conception and design: PZ Liu, XG Shi; (II) Administrative support: XG Shi; (III) Provision of study materials or patients: XM \\ Zuo, X Gao; (IV) Collection and assembly of data: R Lai, Z Zhao; (V) Data analysis and interpretation: PZ Liu, XM Zuo; (VI) Manuscript writing: \\ All authors; (VII) Final approval of manuscript: All authors. \\ Correspondence to: Xiao-Guang Shi. Department of Galactophore, Dongzhimen Hospital of Beijing University of Chinese Medicine, No. 5 of \\ Haiyuncang, Dongcheng District, Beijing, China. Email: shixgtcm915@163.com.
}

\begin{abstract}
Background: The objective of this study was to evaluate the clinical efficacy of the external application of internal expulsion pus-expelling decoction (IEPED) combined with surgery in the treatment of granulomatous lobular mastitis (GLM).

Methods: A total of 110 patients in our hospital with sepsis GLM were randomly divided into two groups: treatment group ( $n=60$, the wound was treated with IEPED) and control group $(n=50$, the wound was not treated with IEPED). We assessed the recurrence, contra lateral breast form, and aesthetic evaluation of the patients in the two groups.

Results: The total effective rates in the patients in the treatment group and the control group were $90 \%$ and $68 \%$, respectively, after the preoperative pretreatment and before radical surgery $(\mathrm{P}<0.05)$. After 10 days of receiving the debridement treatment, the two groups were compared in term of physical signs scores and the difference was statistically significant $(\mathrm{P}<0.05)$. Within one year of the regular follow-up after treatment, 0 case recurred in the treatment group and 1 case recurred in the control group $(\mathrm{P}>0.05)$. In the treatment group, 30 cases showed excellent results in the aesthetic evaluation of breast appearance, 18 cases were good, and the overall excellent and good rate was up to $80 \%$. In the control group, 12 cases showed excellent results and 16 cases showed good results, with the overall excellent and good rate reaching 56\% $(\mathrm{P}<0.05)$.

Conclusions: In patients with abscess debridement of GLM, the external application of IEPED can significantly reduce the primary lesion of patients with abscess GLM, reduce the surgical resection area, and maximize the preservation of the patients' breast appearance.
\end{abstract}

Keywords: Granulomatous lobular mastitis (GLM); internal expulsion pus-expelling decoction (IEPED); external treatment; surgical treatment

Submitted Dec 27, 2019. Accepted for publication Apr 07, 2020.

doi: $10.21037 /$ apm-19-684

View this article at: http://dx.doi.org/10.21037/apm-19-684

\section{Introduction}

Granulomatous lobular mastitis (GLM), also known as granulomatous mastitis, is a granulomatous inflammatory response centered on the lobules of the breast and is characterized by non-caseous necrosis of the breast. In the past, GLM was rare in clinical practice and, in recent years, the incidence of this disease has increased yearly (1). As there is a lack of specificity in the clinical manifestations and imaging examination of this disease and its pathological characteristics have not been fully identified by clinicians, 
it is easy to be misdiagnosed as breast cancer, plasma cell mastitis, breast tuberculosis, and other diseases. Improper treatment of GLM often leads to breast ulceration, and non-recovery of the lesions causes damage to the breast, and may even lead to a total mastectomy, causing a significant psychological burden in patients (2).

At present, the treatment of GLM is mainly surgical resection, which is an effective method to treat the disease (3). However, improper timing of the operation often causes distortion of the breast appearance, affects the attractiveness of the breasts, and aggravates the psychological burden and social barriers in patients (4). Because the disease is often found in young women of childbearing age who have high requirements for breast appearance, it is necessary to choose the correct timing of the operation and preoperative clinical downstaging of the lesions. The clinical stages of GLM include the mass stage, the pus-forming stage, the pus stage, and the sinus stage. A considerable number of patients visit their doctors in the pus-forming stage. Due to the large scope of the lesion in GLM in the pus-forming stage, extensive empyema around the lesion often enlarges the scope of the operation, increases the difficulty of operation, and since the wide dissemination of the lesions leads to the conventional surgical removal of the lesions not being complete, the patients easily relapse after the operation.

In order to investigate the more effective therapy for GLM, we try to research the effectiveness of integrated traditional Chinese and western medicine for the GLM. Internal expulsion pus-expelling decoction (IEPED) comes from the Medical Golden Mirror ancient literature, which has the effects of internal expulsion, detumescence and apocenosis, toning qi, and toning the spleen. Ordinarily, IEPED is mainly used for the treatment of external carbuncle characterized by completed carbuncle and a deficiency of Qi and blood and excess of pathogenic factors with the main usage including taking the treatment orally taking after decoction. In the decoction, pangolins are salty, bitter and cold, its medical power mainly travels along the liver and stomach meridians, it has the effects of stasis-removing, eliminating stagnation, detumescence and removing pus, and is the main drug in the treatment of internal and external carbuncle. Gleditsia has the effects of detumescence, eliminating stagnation, extracting toxins, removing pus, and is used at the beginning of the treatment of skin and external diseases with non-broken pus. As a classical prescription for the treatment of carbuncle without purulence, there are relatively fewer studies on the treatment of GLM in the pus-forming stage by the external application of IEPED. Therefore, the purpose of this study is to assess and evaluate the clinical effect of the external application of IEPED in the treatment of GLM in the pusforming stage.

\section{Methods}

\section{Study subjects}

In the present study, female patients who were diagnosed with GLM by puncture pathology and admitted to our hospital from March 2017 to July 2018 were selected as the study subjects. After completing the B-ultrasound examination, it was revealed that the patients had GLM in the pus-forming stage. According to different treatment methods, these patients were randomly divided into two groups using the random number table method: the treatment group (IEPED external application group) and the control group (non IEPED external application group). The Ethics Committee of our hospital approved this study (approval number:2017-JYB-JS-063) and all patients provided signed informed consent. This study was conducted in accordance with the Declaration of Helsinki (as revised in 2013).

\section{Inclusion and exclusion criteria}

Inclusion criteria: (I) patients with a clear pathological result of GLM by coarse needle biopsy after admission; (II) non lactating mastitis patients with an age of 23-37 years old; (III) GLM patients who were suspected to be in the pus-forming stage, B-ultrasound examination revealed that large flaky low echo areas could be seen in the subcutaneous space on the affected side of the breast, the shape was not regular, and the boundary was not clear.

Exclusion criteria: (I) pregnant women; (II) patients with severe heart, liver, and kidney dysfunctions; (III) patients with coagulation dysfunction; (IV) patients with allergies or other adverse reactions after the external application of IEPED on the lesions after admission; and (V) patients who received follow-ups less than one year after the end of the treatment.

\section{Research methods}

In the treatment group, after completing the clinical physical examination and/or B-ultrasonic examination, 
the patients were found to have breast masses and pus formation; the patients then underwent abscess debridement after the operation, received anesthesia and the exclusion of contraindications, and were treated with $1 \%$ lidocaine for local anesthesia according to the conditions before debridement. The most fluctuating part was used as the puncture point and an approximate incision of $0.5 \mathrm{~cm}$ long was made, the necrotic purulent cavity in the gland tissue was fully opened with tissue forceps, the necrotic tissue and edema granulation in the breast were removed, residual pus and congestion was sucked out with an aspirator; the residual cavity was fully washed with iodophor water and hydrogen peroxide, and then the residual cavity was wetdressed with IEPED and bound up. The composition of the basic prescription of IEPED: $12 \mathrm{~g}$ of atractylodes macrocephala, $12 \mathrm{~g}$ of pangolin, $12 \mathrm{~g}$ of ginseng, $12 \mathrm{~g}$ of angelica, $9 \mathrm{~g}$ of cohosh, $9 \mathrm{~g}$ of licorice, $15 \mathrm{~g}$ of angelica, $15 \mathrm{~g}$ of Astragalus, $10 \mathrm{~g}$ of Gleditsia, and $10 \mathrm{~g}$ of green tangerine peel. Usage: all drugs were deeply fried with water until the volume reduced $100 \mathrm{~mL}$ and then the liquid medicine was cooled to normal temperature when used externally, the sterile gauze was soaked and then filled in the purulent cavity. For patients with obvious bleeding, a piece of Vaseline gauze was filled in the purulent cavity to stop the bleeding on the day after debridement, which was taken out after the bleeding was stopped on the next day. After debridement, the dressing was changed 1-2 times a day according to the condition of the wound and, after each dressing change, the affected area was wet-dressed with IEPED and bound up. The therapeutic effect was evaluated 10 days after treatment and the conditions of the patients were re-evaluated and then the operation was performed.

In the control group, patients underwent abscess debridement similarly after the disease was confirmed and were treated with $1 \%$ lidocaine for local anesthesia according to the condition before debridement. The most fluctuating part was used as the puncture point and an approximate incision of $0.5 \mathrm{~cm}$ long was made. The necrotic purulent cavity in the gland tissue was fully opened with tissue forceps, the necrotic tissue and edema granulation in the breast were removed, residual pus and congestion was sucked out with an aspirator; the residual cavity was fully washed with iodophor water and hydrogen peroxide, and then the residual cavity was only filled with an ordinary gauze and bound up. After debridement, the dressing was changed 1-2 times a day according to the condition of the wound. The therapeutic effect was evaluated 10 days after treatment and the conditions of the patients were re- evaluated and then the operation was performed.

During the treatment, the patients in the two groups received the same kinds of antibiotic anti-inflammatory therapy after surgery: an intravenous drip of $1.5 \mathrm{~g} /$ time cefuroxime sodium injection, bid (patients allergic to cefuroxime received an intravenous drip of $300 \mathrm{mg} / \mathrm{time}$ etimicin sulfate, qd). The course of treatment was 7-14 days.

The patients in the two groups were treated with the above mentioned preoperative comprehensive pretreatment measures, were re-evaluated for their conditions, and then received the operation after 10 days of treatment. The principle of the operation was that, with the inflammatory lesions as the center, the surgical incisions were made in different sites according to the location of the masses, the local skin ulceration or areola was mainly chosen. Firstly, the lesions and the normal glands and adipose tissue within $1 \mathrm{~cm}$ around the lesions were completely removed, then an exploration of the mammary ducts of the affected side of the breast was performed. The local mammary ducts with lesions were removed and patients presenting an inverted nipple on the affected side underwent papilloplasty to correct the inverted nipple and prevent the recurrence of the disease. After the thorough removal of the lesions, the surgical field was washed repeatedly with iodophor water (normal content of iodophor diluted with normal saline) and normal saline, then, the free gland flap displacement was performed to reconstruct the appearance of the breast and a drainage tube was placed to prevent the formation of effusion. After the operation, the change in the drainage volume in the operation area was observed, until the drainage volume was less than $30 \mathrm{ml}$ for three consecutive days and no subcutaneous effusion was found in the B-ultrasonic examination, the drainage tube was removed. According to the wound healing condition, the dressing was changed timely. At 12-14 days after the operation, stitch removal was performed and after the stitch removal, if the patients did not complain about experiencing unusual discomfort and no abnormality was found in physical examination, the patients were allowed to be discharged and underwent a regular reexamination.

\section{The main observation indexes}

\section{Classification of signs}

Based on relevant literature (5), the classification system of signs of GLM was established: the lesion range was divided into three grades: (I) grade 0: no lesion is found 
by ultrasound; grade 1: the scope of the lesions found by ultrasound was $<4 \mathrm{~cm}^{2}$; grade 2 : the scope of the lesions found by ultrasound was $\geq 4$ and $\leq 25 \mathrm{~cm}^{2}$; grade 3 : the scope of the lesions found by ultrasound was $\geq 25 \mathrm{~cm}^{2}$. (II) Local skin color: grade 0: normal; grade 1: red. (III) Abscess formation: grade 0: no; grade 1: yes. (IV) Formation of sinus or fistula: grade 0: no; grade 1: yes. (V) Nipple discharge: grade 0: no; grade 1: yes. (VI) Axillary lymphadenectasis: grade 0: no; grade 1: yes. (VII) Formation of nodular erythema of the lower limbs: grade 0: no; grade 1: yes. (VIII) Free joint pain in the whole body: grade 0: no; grade 1: yes. The score corresponding to the classification is the following: grade 0: 0 point; grade 1: 2 points; grade 2: 4 points; grade 3: 6 points. The scores of signs before debridement and at 10 days after debridement were recorded and compared between the two groups.

\section{Clinical effect}

In the present study, the clinical effect was compared between the two groups according to the actual clinical situation on the 10th day after the abscess debridement of the affected side of the breast. Before the operation, according to the actual situation of treatment, the clinical treatment effects of all patients were divided into three levels. Excellent: there is redness and swelling on the superficial layer of the breast surface, the pain is obviously relieved, and the mass is obviously reduced; the mass shrinks by more than $1 / 3$ and the exudation of the wound is significantly less. Effective: there is redness and swelling on the skin of the breast surface, the pain is slightly relieved, and the mass is reduced; the mass shrinks by less than $1 / 3$ and the exudation of the wound is less or slightly less. Ineffective: the symptoms of redness, swelling, hotness, and pain of the breast are not improved significantly and the volume of the mass remains unchanged; the wound still exudes a large amount of pus and even the scope of the lesion is enlarged, and the disease is aggravated. Total effective rate $=$ (effective cases + effective cases)/total cases $\times 100 \%$.

\section{Aesthetic evaluation}

Half a year after the operation, the aesthetic evaluation was made on the breast appearance of the two groups. According to Harris standard developed in 2001, the Evaluation Form of Breast Preservation Aesthetics and Breast Aesthetics Viewpoint formulated in the national "The Tenth Five Year Plan" scientific and technological research project (2001BA7303B20) and clinical treatment experience was used to summarize the following bilateral breast symmetry items. The specific evaluation criteria are presented in Table 1.

\section{Postoperative follow-up}

After treatment, all patients received follow-ups by telephone and the number of recurrence cases in all groups was recorded. Six months after the operation, the patient was asked to return to the operation hospital for re-examination and the aesthetic evaluation of the affected side of the breast was carried out to evaluate the long-term effect. The patients were reexamined regularly at 3, 6, 9, and 12 months after the operation and the time of the reexamination was the same as that of the telephone follow-ups.

\section{Statistical analysis}

In the present study, data were analyzed using statistical software SPSS20.0. Measurement data were expressed as mean \pm standard deviation $(\bar{x} \pm S D)$. Count data were expressed as a percentage (\%). The test of normality was conducted using a $W$-test and the homogeneity of variance was tested using an F-test. Inter-group comparisons were conducted using a $t$-test. Non-normally distributed means of multiple samples or normally distributed means of multiple samples with heterogeneity of variance were compared using a nonparametric test. Count data were compared using a Chi-square test. $\mathrm{P}<0.05$ was considered statistically significant.

\section{Results}

\section{General data}

In the present study, 110 female patients with GLM that was accurately diagnosed by biopsy pathology were included. These patients were randomly divided into two groups: treatment group $(n=60$, IEPED external application group) and control group $(n=50$, non IEPED external application group). In the treatment group, the age of these patients ranged within 23-37 years old, with an average age of $30.40 \pm 3.43$ years old. In the control group, the age of these patients ranged within 25-37 years old, with an average age of $30.72 \pm 2.701$ years old. All patients only had a unilateral lesion. In the treatment group, 26 patients had lesions on the left side and 34 patients had lesions on the right side; in the control group, 22 patients had lesions on the left side and 28 patients had lesions on the right side. No patient was found to have bilateral lesions. All patients 
Table 1 Aesthetic evaluation of breast

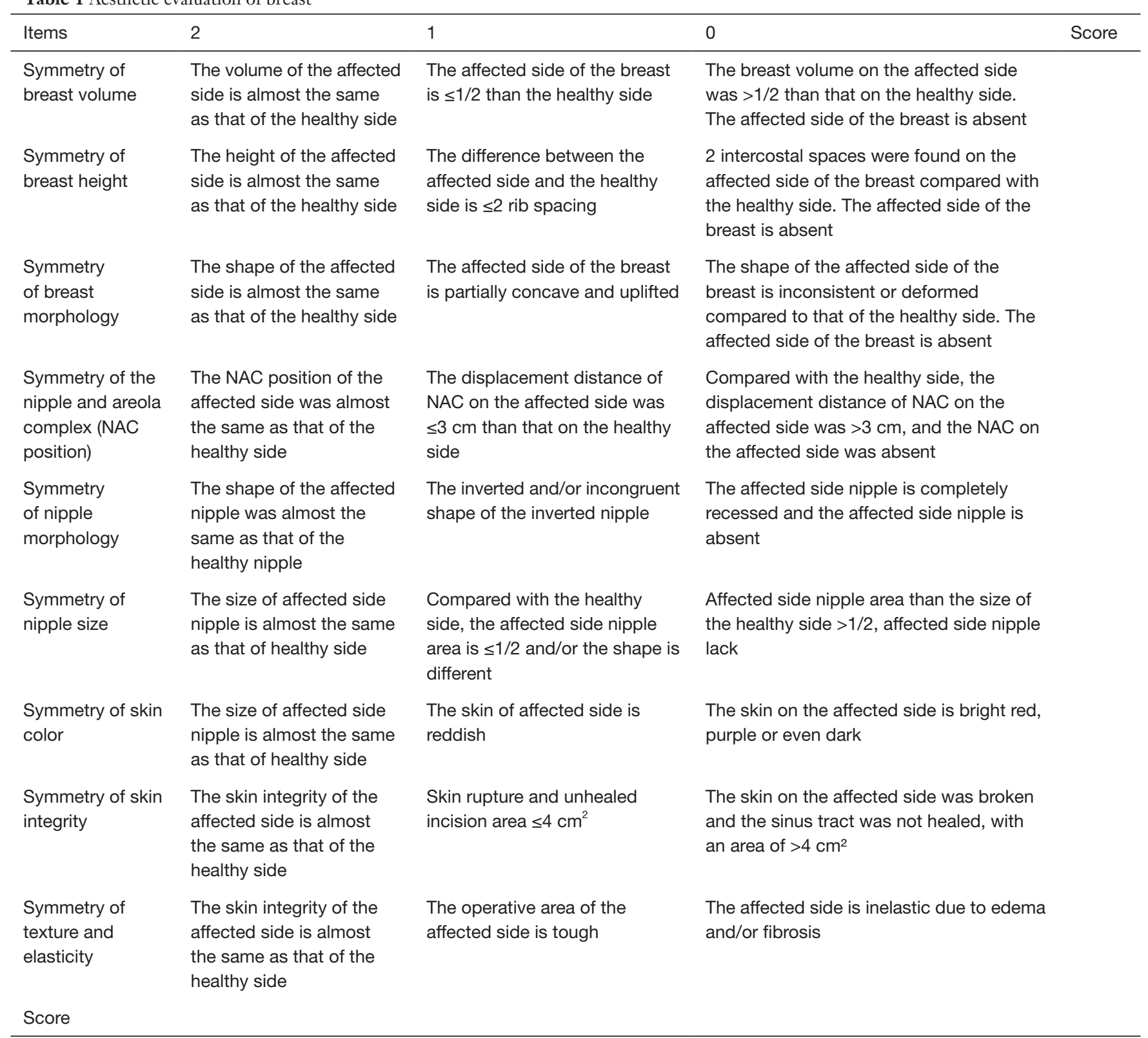

Scoring criteria: the total score is 18 points, the total score $\geq 14$ is excellent, the total score $\leq 12$ is good, and the total score $\leq 8$ is average.

A total integral less than 8 is a difference.

were married and had children. The differences in the baseline data between the two groups were not statistically significant $(\mathrm{P}>0.05)$, so the two groups were comparable.

\section{Comparison of age and sign scores}

\section{Age and sign scores at admission}

The difference in the mean age between the treatment group and the control group was not statistically significant $(\mathrm{P}>0.05)$. Before debridement and after admission, the average score of signs was $12.27 \pm 2.08$ and $12.64 \pm 2.14$ in the treatment group and control group, respectively $(\mathrm{P}=0.546)$. The differences in the mean age and physical signs before treatment between the two groups were not statistically significant $(\mathrm{P}>0.05)$, so the two groups were comparable (Table 2). 


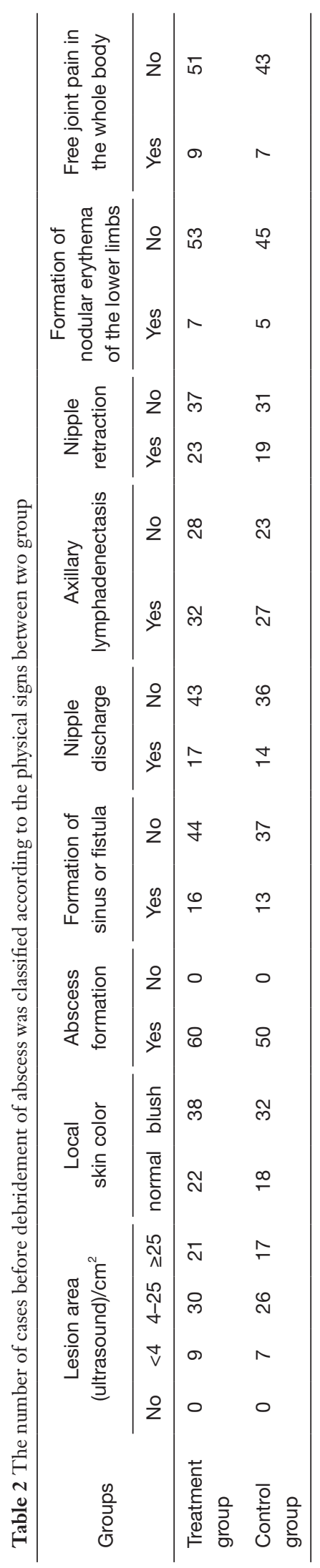

Scores of signs at $\mathbf{1 0}$ days after debridement

At 10 days after debridement, the average sign scores were $6.93 \pm 1.72$ and $9.68 \pm 2.36$, respectively, in the treatment group and the control group and the sign scores in the two groups were lower at 10 days after treatment than before treatment. The sign score was also lower in the treatment group than in the control group $(\mathrm{P}=0.001)$, the difference was statistically significant $(\mathrm{P}<0.01$, Table 3).

\section{Comparison of the clinical effect}

\section{Clinical effective rate}

An accurate Chi-square test revealed that, at 10 days after debridement, the differences in the total number of effective cases and the ineffective cases between the two groups were statistically significant $(\mathrm{P}<0.05)$. In the treatment group, after 10 days of the external application of traditional Chinese medicine on the wound, 28 patients achieved an excellent effect, 26 patients achieved an effective result, and 6 patients suffered from an ineffective result, the total effective rate was $90.00 \%$ (54/60); in the control group, after 10 days of applying the conventional dressing change, 7 patients achieved an excellent effect, 27 patients achieved an effective result, and 16 patients suffered from an ineffective result, the total effective rate was $68.00 \%$ (34/50). The difference between the two groups was statistically significant $(\mathrm{P}=0.02,<0.05)$ and the clinical effect was better in the treatment group than in the control group (Table 4).

\section{Aesthetic evaluation of the patients' breasts after the operation}

The aesthetic appearance of the breasts in the control group and the treatment group was compared and the results were as follows: The aesthetic scores were $13.33 \pm 1.45$ in the treatment group and $12.16 \pm 2.09$ in the control group. The aesthetic scores of the treatment group were higher than those of the control group $(\mathrm{P}=0.038)$, the difference was statistically significant $(\mathrm{P}<0.05$, Table 5$)$.

\section{Postoperative recurrence rate}

A chi-square test revealed that within one year of the regular follow-ups after treatment, the number of recurrence cases in the two groups was compared: in the treatment group, 0 patient experienced a recurrence and the recurrence rate was $0 \%(0 / 60)$; in the control group, 1 patient experienced a recurrence and the recurrence rate was $2 \%(1 / 50)$. The 
Table 3 Scores of signs at 10 days after debridement between two groups

\begin{tabular}{lcccc}
\hline Index & Total $(\mathrm{n})$ & Treatment group & Control group & $\mathrm{P}$ \\
\hline Before treatment & 60 & $12.27 \pm 2.08$ & $12.64 \pm 2.14$ & 0.546 \\
After treatment & 50 & $6.93 \pm 1.72$ & $9.68 \pm 2.36$ & 0.001 \\
\hline
\end{tabular}

Table 4 The effective between two groups at 10 days after debridement

\begin{tabular}{|c|c|c|c|c|c|}
\hline Groups & Excellent effect & Effective & Ineffective & Total effective rate (\%) & $P$ \\
\hline Control group & 7 & 27 & 16 & $68.00 \%$ & \\
\hline
\end{tabular}

Table 5 Aesthetic evaluation of the patients' breasts after the operation between two groups

\begin{tabular}{lccccccc}
\hline Index & $\mathrm{n}$ & Excellent & Good & General & Bad & Z & $P$ \\
\hline Treatment group & 60 & 30 & 18 & 12 & 0 & -2.075 \\
Control group & 50 & 12 & 16 & 22 & 0 & 0.038 \\
\hline
\end{tabular}
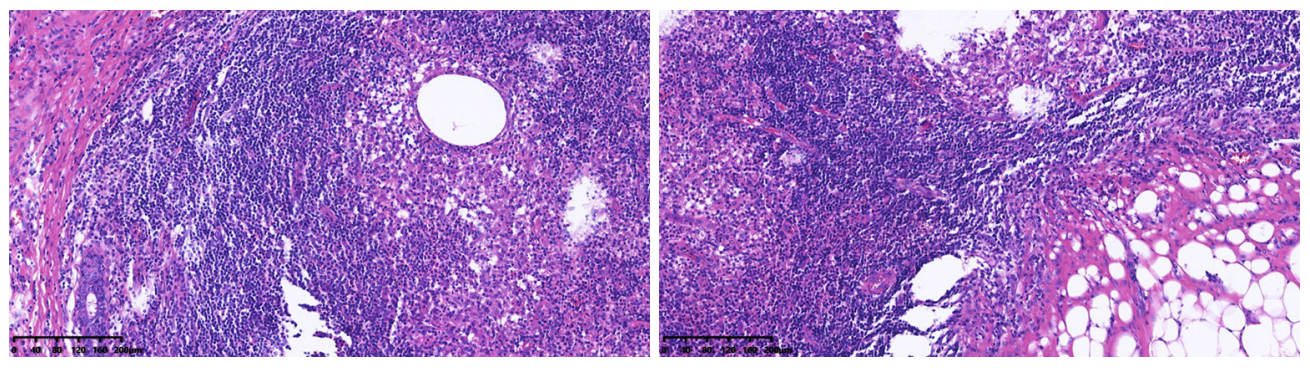

Figure 1 Typical pathological manifestations of GLM: (surgically removed breast gland tissues) severe acute and chronic inflammation of the breast with a small abscess and formation of foreign body granuloma, some of them are granulomatous lobular mastitis, some areas of the lesions are yellow granulomatous mastitis, mammary gland disease; chronic inflammation of the skin tissue, it can be seen that the epidermis falls into the deep layer locally. Staining: H\&E 20x. (Left breast gland tissue) breast severe acute and chronic inflammation with abscess and cyst cavity formation, the wall can be seen necrosis with inflammatory granulation tissue formation, local wall fibrosis, part of the lobular granulomatous mastitis, breast Adenopathy with cystic hyperplasia, papillary hyperplasia with bridge hyperplasia, local cell vacuolar degeneration, some duct cystic expansion, some fat necrosis with chronic inflammation and fibrosis. (Pathology photo from Beijing University of Chinese Medicine Department of Pathology, Dongzhimen Hospital). GLM, granulomatous lobular mastitis.

difference in the postoperative recurrence rate between the two groups was not statistically significant $(\mathrm{P}=0.271,>0.05$, Figures 1-3).

\section{Discussion}

The results of this study revealed that the differences in age and signs before treatment between the two groups were not statistically significant. In the treatment group, the total effective rate was $90 \%$ and in the control group, the total effective rate was $68 \%$, the difference in the total effective rate between the two groups was statistically significant. After 10 days of debridement treatment, the difference in the sign scores between the two groups was statistically significant. Within one year of regular followups after treatment, the number of recurrence cases in the two groups was compared: in the treatment group, 0 patients experienced a recurrence and the recurrence 

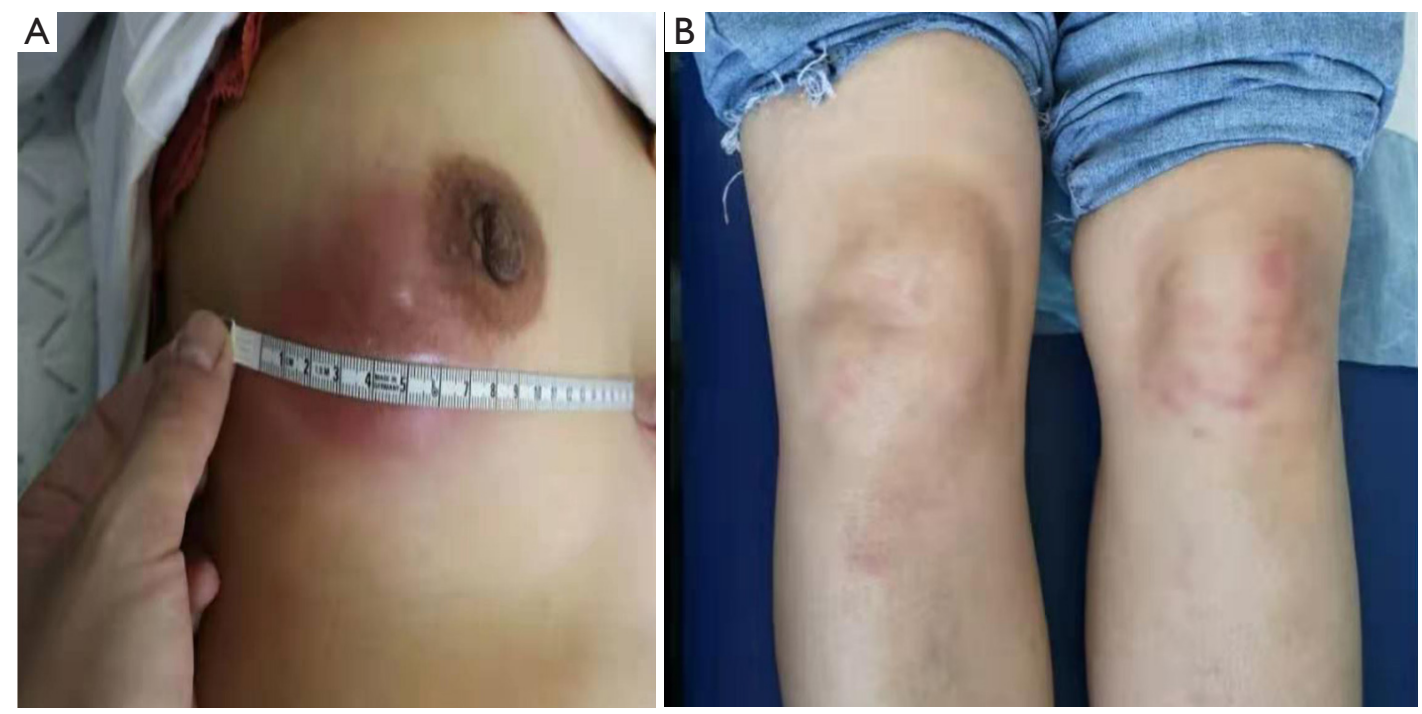

Figure 2 Typical clinical signs of GLM. (A) Extensive redness and swelling in the outer lower quadrant with massive subcutaneous empyema of the right breast; (B) erythema nodosum of both lower limbs. GLM, granulomatous lobular mastitis.
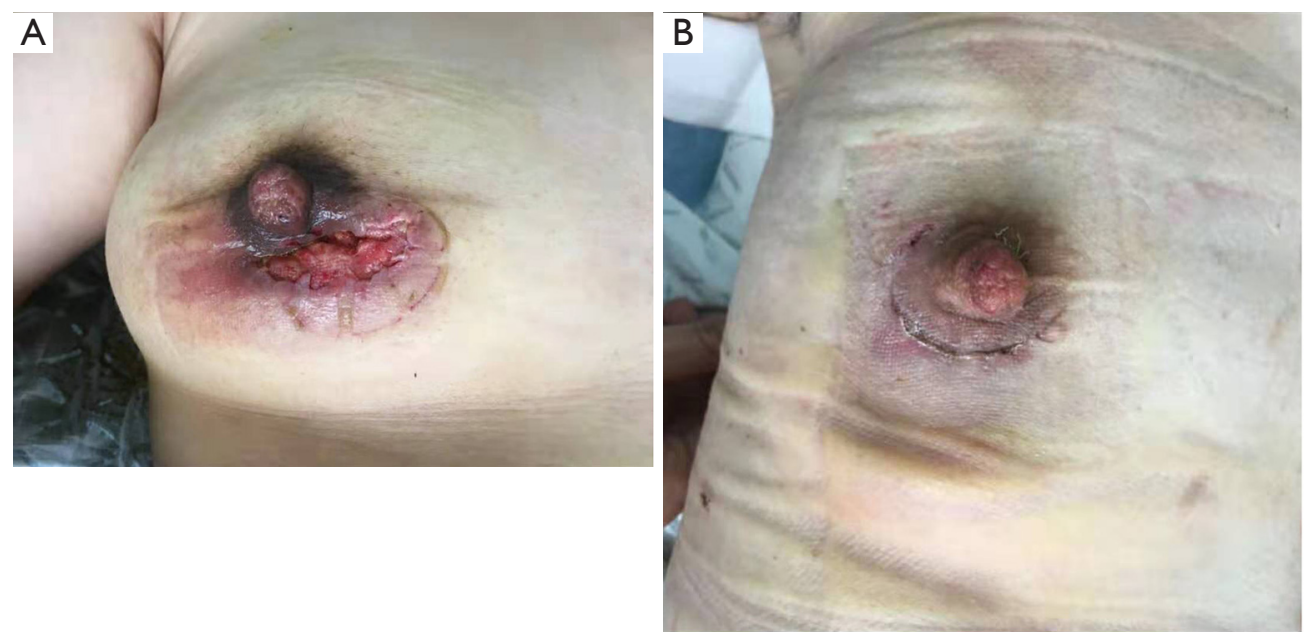

Figure 3 Treatment methods of GLM. (A) Incision and drainage of abscess + debridement after admission; (B) radical operation after the reduction of lesions (12 days after the operation). GLM, granulomatous lobular mastitis.

rate was $0 \%$; in the control group, 1 patient experienced a recurrence and the recurrence rate was $2 \%$; the difference was not statistically significant. Half a year after the operation, the aesthetic evaluation was made on the breast appearance of all followed-up patients in the two groups. In the treatment group, 30 patients had excellent results and 18 patients had good results in the aesthetic evaluation of the breast appearance, the overall excellent rate was $80 \%$; in the control group, 12 patients had excellent results and 16 patients had good results, the overall excellent rate was
$56 \%$; the difference between the two groups was statistically significant.

GLM, also known as idiopathic granulomatous mastitis (IGM), is a rare, non-lactation chronic mastitis disease with an unknown etiology. The pathological feature is granulomatous inflammation with the lobule as the center. Its main components are epithelioid cells, multinucleated giant cells, neutrophils, etc. Micro abscess formation and non-caseous necrosis in the lesion area are one of a variety of characteristics of granulomatous mastitis (GM). This 
disease was first reported by Kessler in 1972 (6) and first reported in China by Ma in 1986 (7). In the past, because the disease was rare, there was no single classification method of the disease in the ancient literature of traditional Chinese medicine until the 1980s when Bohua Gu, Deming Lu, and Hanjun Tang et al. summarized the clinical characteristics of the disease and named it "acne mastitis" and included the disease in the Practical Surgery of Traditional Chinese Medicine, edited by Mr. Bohua Gu for the first time (8). In the past, the incidence of the disease was not high, therefore, there are still many breast doctors who lack accurate knowledge of the disease. The disease is also often misdiagnosed as breast cancer, suppurative mastitis, plasma cell mastitis, and other breast-related diseases, resulting in a delay in treatment. However, the incidence of GLM has increased rapidly in recent years and is significantly higher than the incidence of plasma cell mastitis and mammary duct fistula plasma cell mastitis. Previous literature reported that GLM may be related to pregnancy, hyperprolactinemia, lactation disorder, trauma, Corynebacterium infection, $\alpha$ 1-antitrypsin deficiency, dietary pollution, oral contraceptives or other drugs (9-13). Most scholars believe that the histologic manifestations of this disease are similar to granulomatous thyroiditis and granulomatous orchitis and since no pathogenic bacteria can be found in the lesion, it is an autoimmune disease (14). Brown et al. revealed in their studies that (15) this disease may be a local immune phenomenon and a hypersensitivity reaction caused by milk and belongs to the type IV local delayed allergy of the breast.

Since most patients lose the opportunity of early conservative treatment, most GLM patients finally choose to undergo surgery (14,16-19). Proper operation can completely remove the granulation in the lesions and the long-term effect is reliable. However, if the scope of preoperative lesions is wide and it is accompanied by purulent ulceration, during the operation, the surgeon should not only completely remove the lesions but also simultaneously consider the appearance of the breast and then it becomes difficult to operate. The conventional western medicine treatment view is that the inflammatory phase is the relative contraindication of surgery and it is easy to extend the lesions and aggravate the infection; however, allowing the lesions to expand without surgical treatment may lead to further progress of the disease and increase the postoperative recurrence rate. Therefore, how to reduce the scope of GLM lesions before the operation and create opportunities for a radical operation has always been the goal of unremitting efforts of breast surgeons. The results of many studies revealed that integrated traditional Chinese and western medicine has unique advantages in the treatment of GLM. The purpose of this study was to assess the advantages of traditional Chinese medicine combined with surgery in the treatment of the pus-forming stage of GLM.

The purpose of this study was also to assess the feasibility and clinical effect of the external application of traditional Chinese medicine in the treatment of the pusforming stage of GLM.A previous study revealed that traditional Chinese medicine has unique advantages in the treatment of GLM. In this study, the classic prescription of IEPED was used to treat GLM by the external application on the wound. IEPED comes from the Medical Golden Mirror ancient literature, which has the effects of internal expulsion, detumescence and apocenosis, toning qi, and toning the spleen. Ordinarily, IEPED is mainly used for the treatment of external carbuncle characterized by completed carbuncle and a deficiency of Qi and blood and excess of pathogenic factors with the main usage including the treatment by orally taking decoction. In the decoction, pangolins are salty, bitter and cold, its medical power mainly travels along the liver and stomach meridians, it has the effects of stasis-removing, eliminating stagnation, detumescence and removing pus, and is the main drug in the treatment of internal and external carbuncle. Gleditsia has the effects of detumescence, eliminating stagnation, extracting toxins, removing pus, and is used at the beginning of the treatment of skin and external diseases with non-broken pus. "Collected Statements on the Herbal Foundation" states that: "Gleditsia, pulls toxin and dispels wind for patients with incomplete carbuncle and it can force the lesion to dissipate. For those lesions that are going to break, it can force them to dissipate." Its effects include detumescence and removing toxins and pus. Using both methods has the effects of detumescence, eliminating stagnation, and removing toxins and pus. In the decoction, ginseng and Astragalus have the effects of toniyng qi, tonying the spleen, internal expulsion, and removing toxins; cohosh has the effects of breaking anthema, detoxifying, invigorating splenic yang, and lifting prolapsed organs; angelica has the effects of bloodactivating, removing pus, promoting granulation, and relieving pain. The combined use of these drugs has the effects of clearing heat, detumescence, and removing toxins and pus. IEPED can reduce the inflammatory response of local tissues, improve local microcirculation, 
relieve local symptoms, and shorten the time of wound healing. In the present study, IEPED was used for the external application on the wounds and the preoperative total effective rate was up to $90.00 \%$, significantly better than the simple routine dressing change treatment in the control group (with a total effective rate of $68.00 \%$ ). However, there was no statistical significance in reducing the recurrence rate $(\mathrm{P}=0.271)$, which still needs further analysis to confirm the effects. This clinical trial revealed that the external application of traditional Chinese medicine on the wound after abscess debridement can control the type IV allergic reaction of local lesions, reduce the scope of lesions, reduce the scope of surgical exploration and resection, reduce the damage of breast glands caused by the operation, and maintain the breast appearance.

This study still has the following limitations. Firstly, the present study was a clinical randomized controlled study but it was not blinded, therefore, there is still a certain risk of bias. Secondly, this study is a single-center clinical trial, the included sample size is small, and multi-center clinical trials with a larger sample size are still needed. Thirdly, the clinical follow-up time of this study was short and further clinical follow-ups are still needed. Finally, at present, the exact cause of GLM is not clear, the best treatment wellrecognized by the academic community has not been unified, there is still great controversy on the standardized treatment of GLM, and further research is needed. Fifthly, the possible mechanism of IEPED maybe related to the inhibition of inflammation which should be further research by an animal trial in the future.

\section{Conclusions}

The external application of IEPED on the wound surface of patients with GLM in the pus-forming stage after debridement has certain advantages in alleviating the patients' condition, reducing the scope of the operation, and maximizing the preservation of the breast appearance and is worthy of clinical popularization and application.

\section{Acknowledgments}

Funding: Beijing university of traditional Chinese medicine 2017 basic scientific research project book (Young teacher project). Project name: the effect of tori pyodialysis on serum IgG4, CD4, CD8, RF, Clq in patients with granulomatous lobular mastitis in abscess stage.

\section{Footnote}

Conflicts of Interest: All authors have completed the ICMJE uniform disclosure form (available at http://dx.doi. org/10.21037/apm-19-684). The authors have no conflicts of interest to declare.

Ethical Statement: The authors are accountable for all aspects of the work in ensuring that questions related to the accuracy or integrity of any part of the work are appropriately investigated and resolved. The study was approved by institutional ethics committee of Dongzhimen Hospital of Beijing University of Chinese Medicine (No. 2017-JYB-JS-063) and conducted in accordance with the Declaration of Helsinki (as revised in 2013). All patients provided signed informed consent.

Open Access Statement: This is an Open Access article distributed in accordance with the Creative Commons Attribution-NonCommercial-NoDerivs 4.0 International License (CC BY-NC-ND 4.0), which permits the noncommercial replication and distribution of the article with the strict proviso that no changes or edits are made and the original work is properly cited (including links to both the formal publication through the relevant DOI and the license). See: https://creativecommons.org/licenses/by-nc-nd/4.0/.

\section{References}

1. Kok KY, Telisinghe PU. Granulomatous mastitis: presentation, treatment and outcome in 43 patients. Surgeon 2010;8:197-201.

2. Zhou F, Yu LX, Ma ZB, et al. Granulomatous lobular mastitis. Chronic Dis Transl Med 2016;2:17-21.

3. Brownson KE, Bertoni DM, Lannin DR, et al. Granulomatous lobular mastitis- Another paradigm shift in treatment. Breast J 2019;25:790-1.

4. Chen L, Zhang XY, Wang YW, et al. Granulomatous lobular mastitis: a clinicopathological analysis of 300 cases. Zhonghua Bing Li Xue Za Zhi 2019;48:231-6.

5. Liu L, Zhou F, Zhang X, et al. Granulomatous Lobular Mastitis: Antituberculous Treatment and Outcome in 22 Patients. Breast Care (Basel) 2018;13:359-63.

6. Kessler E,Wolloch Y. Granulomatous mastitis-lesion clinically simulating carcinoma. Am J Clin Pathol 1972;58:642-6.

7. Ma GH, Fu XL. Report of 6 cases of breast granuloma. Clinical Oncology of China 1986;13:292. 
8. Gu BH. Practical Chinese surgery. Shanghai: Shanghai Science and Technology Press, 1985.

9. Patel RA, Strickland P, Sankara IR, et al. Idiopathic grannulomatous mastitis: case reports and review of literature. J Gen Intern Med 2010;25:270-3.

10. Lin CH, Hsu CW, Tsao TY, et al. Idiopathic grannulomatous mastitis associated with risperidoneinduced hyperprolactinemia. Diagn Pathol 2012;7:2.

11. Pereira FA, Mudgil AV, Macias ES, et al. Idiopathic granulomatous lobular mastitis. Int J Dermatol 2012;51:142-51.

12. Gurleyik G, Aktekin A, Aker F, et al. Medical and surgical treatment of Idiopathic granulomatous lobular mastitis: a benign inflammatory disease mimicking invasive carcinoma. J Breast Cancer 2012;15:119-23.

13. Özşen M, Tolunay Ş, Gökgöz MŞ. Granulomatous Lobular Mastitis: Clinicopathologic Presentation of 90 Cases Turk Patoloji Derg 2018;34:215-9.

14. Shin YD, Park SS, Song YJ, et al. Is surgical excision

Cite this article as: Liu PZ, Shi XG, Zuo XM, Lai R, Zhao Z, Yang ZR, Liu JL. A clinical study on the treatment of granulomatous lobular mastitis by the external application of the internal pus-expelling decoction and operation. Ann Palliat Med 2020;9(5):2631-2641. doi: 10.21037/apm-19-684 necessary for the treatment of Granulomatous lobular mastitis? BMC Womens Health 2017;17:49.

15. Brown KL, Tang PH. Postlactational tumoral granulomatous mastitis: a localized immune phenomenon. Am J Surg 1979;138:326-9.

16. Kuba S, Yamauchi J, Ohtani H, et al. Vacuum-assisted biopsy and steroid therapy for granulomatous lobular mastitis: report of three cases. Surg Today 2009;39:695-9.

17. Hur SM, Cho DH, Lee SK, et al. Experience of treatment of patients with granulomatous lobularmastitis. J Korean Surg Soc 2013;85:1-6.

18. Gurleyik G, Aktekin A, Aker F, et al. Medical and surgical treatment of idiopathic granulomatouslobular mastitis: a benign inflammatory disease mimicking invasive carcinoma. J Breast Cancer 2012;15:119-23.

19. Erozgen F, Ersoy YE, Akaydin M, et al Corticosteroid treatment and timing of surgery in idiopathic granulomatous mastitis confusing with breast carcinoma. Breast Cancer Res Treat 2010;123:447-52. 